Research Article

\title{
Estimates of DNA damage by the comet assay in the direct-developing frog Eleutherodactylus johnstonei (Anura, Eleutherodactylidae)
}

\author{
Laura Carolina Valencia ${ }^{1}$, Adriana García ${ }^{1}$, Martha Patricia Ramírez-Pinilla ${ }^{2}$ and Jorge Luis Fuentes ${ }^{1}$ \\ ${ }^{1}$ Laboratorio de Microbiología y Mutagénesis Ambiental, Escuela de Biología, \\ Universidad Industrial de Santander, Bucaramanga, Colombia. \\ ${ }^{2}$ Laboratorio de Biología Reproductiva de Vertebrados, Escuela de Biología, \\ Universidad Industrial de Santander, Bucaramanga, Colombia.
}

\begin{abstract}
The aim of this study was to use the Comet assay to assess genetic damage in the direct-developing frog Eleutherodactylus johnstonei. A DNA diffusion assay was used to evaluate the effectiveness of alkaline, enzymatic and alkaline/enzymatic treatments for lysing E. johnstonei blood cells and to determine the amount of DNA strand breakage associated with apoptosis and necrosis. Cell sensitivity to the mutagens bleomycin (BLM) and 4-nitroquinoline-1-oxide (4NQO) was also assessed using the Comet assay, as was the assay reproducibility. Alkaline treatment did not lyse the cytoplasmic and nuclear membranes of $E$. johnstonei blood cells, whereas enzymatic digestion with proteinase $\mathrm{K}(40 \mu \mathrm{g} / \mathrm{mL})$ yielded naked nuclei. The contribution of apoptosis and necrosis (assessed by the DNA diffusion assay) to DNA damage was estimated to range from $0 \%$ to $8 \%$. BLM and 4NQO induced DNA damage in $E$. johnstonei blood cells at different concentrations and exposure times. Dose-effect curves with both mutagens were highly reproducible and showed consistently low coefficients of variation ( $C V \leq 10 \%)$. The results are discussed with regard to the potential use of the modified Comet assay for assessing the exposure of $E$. johnstoneito herbicides in ecotoxicological studies.
\end{abstract}

Key words: bleomycin, Comet assay, DNA diffusion assay, Eleutherodactylus johnstonei, 4-nitroquinoline-1-oxide.

Received: April 29, 2011; Accepted: July 13, 2011.

\section{Introduction}

Amphibians are useful biological indicators because their sensitive skins and occurrence in aquatic and terrestrial habitats makes them vulnerable to environmental change (Blaustein and Wake, 1990; Lips, 1998). Studies using frogs as models to measure the environmental impact of xenobiotics have used mainly larval phases of biphasic frog species such as Anaxyrus americanus, Hyla versicolor, Lithobates catesbeianus, Lithobates clamitans and Lithobates pipiens (Berrill et al., 1994; Relyea, 2004a,b). Most Neotropical frogs have direct development and reproduce on land (Hedges et al., 2008). However, few studies have assessed the usefulness of these species as environmental bioindicators.

Eleutherodactylus johnstonei (Anura: Eleutherodactylidae) is a direct-developing frog (Hedges et al., 2008) from the Lesser Antilles with recently established populations in several Caribbean islands (Kaiser, 1997), as well as in Central and South America (Ortega et al., 2001; Kaiser et

Send correspondence to Jorge Luis Fuentes. Laboratorio de Microbiología y Mutagénesis Ambiental, Escuela de Biología, Universidad Industrial de Santander, A.A. 678, Bucaramanga, Colombia. E-mail: jfuentes@uis.edu.co; jlfuentes2005@yahoo.es. al., 2002). As a consequence of its widespread distribution and certain life history features (reproductive flexibility and high environmental adaptability) (Ortega et al., 2005), E. jonhstonei has been considered a successful invasive species (Rödder, 2009). These attributes also suggest that this species could be a useful model for evaluating the genotoxicological impact of environmental xenobiotics such as pesticides.

DNA damage by environmental xenobiotics is frequently assessed by single cell gel electrophoresis (SCGE) or the Comet assay (Singh et al., 1988), which detects DNA strand breakage and alkali-labile sites by measuring the migration of DNA from immobilized individual cell nuclei. In this assay, the cells are embedded in agarose gel on microscopic slides, lysed and then electrophoresed under alkaline condition. Cells with damaged DNA show increased migration of DNA fragments from the nucleus and the length of the migration indicates the amount of DNA strand breakage; the latter can be estimated by manual and computerized image scoring procedures (Kumaravel et al., 2009). The technique is highly sensitive for detecting DNA damage in any eukaryotic cell type and requires only a few cells. The minimal technical requirements for doing this as- 
say in vitro and in vivo are well established (Cotelle and Férard, 1999; Tice et al., 2000; Hartmann et al., 2003). The Comet assay is sufficiently sensitive for detecting DNA damage in frogs (Dhawan et al., 2009).

The main aim of this work was to assess the usefulness of the Comet assay for detecting DNA damage in $E$. johnstonei. The efficiency of alkaline, enzymatic and alkaline/enzymatic treatments for lysing E. johnstonei blood cells was also assessed using a DNA diffusion assay. Since positive Comet results do not necessarily reflect genotoxicity because DNA strand breakage may be associated with cellular apoptosis and necrosis, we used the DNA diffusion assay (Singh, 2000a) to determine the percentage of DNA strand breakage associated with apoptosis and necrosis $\left(\% \mathrm{~N}_{\mathrm{Ap} / \mathrm{N}}\right)$ and thereby estimate the proportion of DNA strand breakage that was unrelated to apoptosis and necrosis. Cell sensitivity to the mutagens bleomycin (BLM) and 4-nitroquinoline-1-oxide (4NQO) was examined based on DNA strand breakage detected with the Comet assay (Baohong et al., 2005; Kumaravel and Jha, 2006); the reproducibility of the assay in this species was also addressed.

\section{Materials and Methods}

\section{Chemicals}

Bleomycin (BML), dimethyl sulfoxide (DMSO), phosphotungstic acid, molecular grade and low gel temperature (LGT) agaroses, 4-nitroquinoline-1-oxide (4-NQO), silver nitrate and sodium N-lauryl sarcosine were obtained from Sigma-Aldrich Co. (St. Louis, MO, USA). RNaseand DNase-free proteinase $\mathrm{K}$ and PK buffer were obtained from Promega Co. (Madison, WI, USA). Glycerol was purchased from Merck (Darmstadt, Germany). The other reagents and solvents were obtained from J.T. Baker (Phillipsburg, NJ, USA).

\section{Capture and maintenance of frogs}

Eleutherodactylus johnstonei specimens were captured at several sites in the Bucaramanga metropolitan area (Santander, Colombia). Research and collecting permission was given by the Corporación Regional para la Defensa de la Meseta de Bucaramanga (File PC-0014-2008, Resolution 001368). Specimen sex was determined based on species sexual dimorphism and male calling. The frogs were maintained in glass terrariums at $24 \pm 2{ }^{\circ} \mathrm{C}$ on a $12 \mathrm{~h}$ light/dark photoperiod, in conditions (vegetation, air circulation, humidity, illumination, etc.) that simulated the wild habitat. The frogs were fed flies, crickets, spiders, ants and mosquitoes that were captured on the campus of the Universidad Industrial de Santander (Bucaramanga, Colombia).

\section{Blood sampling, cell counts and exposure to mutagens}

Blood obtained by cardiac puncture of cold-anesthetized frogs was collected in heparinized Eppendorf tubes and placed on ice until assayed. Blood cells were counted in a Neubauer counting chamber and then diluted in $0.9 \%$ $(\mathrm{w} / \mathrm{v}) \mathrm{NaCl}$ solution to a cell density of $3.55 \times 10^{6}$ cells $/ \mathrm{mL}$. Aliquots of blood cells were treated with BLM $(0.6-152.0 \mu \mathrm{g} / \mathrm{mL})$ or 4NQO $(1.9-60.0 \mu \mathrm{M})$ for $2,4,6,10$ and $12 \mathrm{~h}$ (selected on the basis of preliminary experiments). Treatments were done at $6 \pm 2{ }^{\circ} \mathrm{C}$ to minimize basal DNA strand breakage. A negative control $(0.9 \% \mathrm{NaCl}$ solution $)$ was always included in each assay. The experiments were done at least three times.

\section{Estimation of DNA strand breakage in E. johnstonei blood cells}

DNA strand breakage in E. johnstonei blood cells was initially assayed by using the alkaline Comet assay, as described by Singh et al. (1988) but with silver staining. Subsequently, DNA strand breakage was detected by the Comet assay as follows: E. johnstonei blood cells were centrifuged (10,000 rpm) and the pellet suspended in proteinase K solution $(20 \mu \mathrm{L})$ prepared in PK buffer $(50 \mathrm{mM}$ Tris- $\mathrm{HCl}, 10 \mathrm{mM} \mathrm{CaCl}_{2}, \mathrm{pH} 8$ ) at $40 \mu \mathrm{g} / \mathrm{mL}$ (concentration determined empirically). Aliquots $(20 \mu \mathrm{L})$ of the cell suspension were mixed with $75 \mu \mathrm{L}$ of $1 \%$ low melting point agarose and the mixture spread on slides containing a layer of $1.3 \%$ molecular grade agarose. The slides were covered with coverslips and incubated at $6 \pm 2{ }^{\circ} \mathrm{C}$ for the agarose to solidify. After enzymatic lysis and agarose polymerization, the coverslips were removed and the slides were placed in a Comet assay tank (Cleaver Scientific Ltd, UK) containing cold alkaline electrophoresis buffer $(0.3 \mathrm{~N} \mathrm{NaOH}, 1 \mathrm{mM}$ EDTA, pH 13) for $25 \mathrm{~min}$. Electrophoresis was done at $25 \mathrm{~V}$ and current adjusted to $300 \mathrm{~mA}$. The slides were routinely exposed to this current in the dark at $6 \pm 2{ }^{\circ} \mathrm{C}$ for $30 \mathrm{~min}$. After electrophoresis, the slides were placed in a staining tray and covered with a neutralizing buffer $(0.4 \mathrm{M}$ Tris-HCl, $\mathrm{pH}$ 7.5) in the dark for $5 \mathrm{~min}$. Silver staining was done as indicated by Díaz et al. (2009).

DNA damage was expressed as arbitrary units based on the classification of comets into five categories (0-4) proposed by Collins et al. (1997). The total amount of DNA strand breakage was expressed in total arbitrary units $\left(\mathrm{AU}_{\mathrm{T}}\right)$ defined as: $\mathrm{AU}_{\mathrm{T}}=\mathrm{N}_{0} \times 0+\mathrm{N}_{1} \times 1+\mathrm{N}_{2} \times 2+\mathrm{N}_{3} \times 3+$ $\mathrm{N}_{4} \times 4$, where $\mathrm{N}_{i}$ is the number of nuclei scored in each category (Collins, 2002). One hundred cells per slide and two slides per blood sample were analyzed using a Nikon Eclipse E200 microscope and the results from at least three independent experiments were averaged to obtain the $\mathrm{AU}_{\mathrm{T}}$ for each treatment.

For the DNA diffusion assay (Singh, 2000a), the cells were processed in a similar manner to the Comet assay, except that the nuclei were not subjected to electrophoresis. Nuclei with a diameter $>3$ times the mean nuclear diameter were considered apoptotic/necrotic (Nigro et al., 2002). The total number of nuclei and the number of apoptotic/ne- 
crotic nuclei in each field were counted (minimum of 100 fields per slide) and the latter then expressed as a percentage of the former. As in the Comet assay, two slides per blood sample were analyzed and the results of at least three independent experiments were averaged to obtain the percentage of apoptotic/necrotic nuclei $\left(\% \mathrm{~N}_{\mathrm{Ap} / \mathrm{N}}\right)$ for each experiment.

Based on the $\mathrm{AU}_{\mathrm{T}}$ and $\% \mathrm{~N}_{\mathrm{Ap} / \mathrm{N}}$ estimates, the proportion of remaining DNA strand breakages was calculated (in arbitrary units) as:

$$
\mathrm{AU}_{\mathrm{R}}=\mathrm{AU}_{\mathrm{T}}-\frac{\% \mathrm{~N}_{\mathrm{AP} / \mathrm{N}} \times \mathrm{AU}_{\mathrm{T}}}{100}
$$

where $\mathrm{AU}_{\mathrm{R}}$ corresponds to non-apoptotic/necrotic DNA strand breakages.

\section{Statistical analysis}

In all cases, the data passed the Kolmogorov-Smirnov and F-maximum tests for normality and variance homogeneity, respectively, so that parametric tests were used in subsequent data analyses. When a significant F-value was obtained in one-way analysis of variance (ANOVA) the groups were subsequently compared with Students $t$-test. Product-moment (Pearson) correlation analysis was used to examine the relationship between mutagen doses and the average estimates of DNA damage. A value of $p<0.05$ indicated significance. All statistical analyses were done with STATISTICA V.6 software (StatSoft Inc).

\section{Results}

\section{Cellular lysis assays}

In the present work, we used the Comet assay described by Singh et al. (1988) to detect DNA strand breakage in E. johnstonei blood cells. These cells were not lysed by alkaline conditions and naked nuclei were not obtained. Although cells varied in their sensitivity to silver staining (Figure 1), neither the $\mathrm{pH}$ (between 10 and 13) of the incubation solution nor the length of incubation ( $2 \mathrm{~h}$ and $24 \mathrm{~h}$ ) had any effect on the susceptibility to lysis (data not shown).

Since naked nuclei could not be obtained from $E$. johnstonei blood cells by the standard Comet procedure (Singh et al., 1988), we compared the ability of alkaline, enzymatic and alkaline/enzymatic treatments to produce these nuclei, as assessed by the DNA diffusion assay (Singh, 2000a). Treatments that included enzymatic lysis (Figure 2B,C) were effective in producing naked nuclei from blood cells, in contrast to lysis by alkaline treatment (Figure 2A). Combined alkaline/enzymatic treatment (Figure 2B) was more aggressive to nuclear stability than enzymatic treatment (Figure 2C), as shown by the nuclear diameter. The Comet assay showed that alkaline/enzymatic treatment produced DNA damage after a very short exposure to alkaline lysis $\left(\mathrm{AU}_{5} \min =295 \pm 11\right.$,

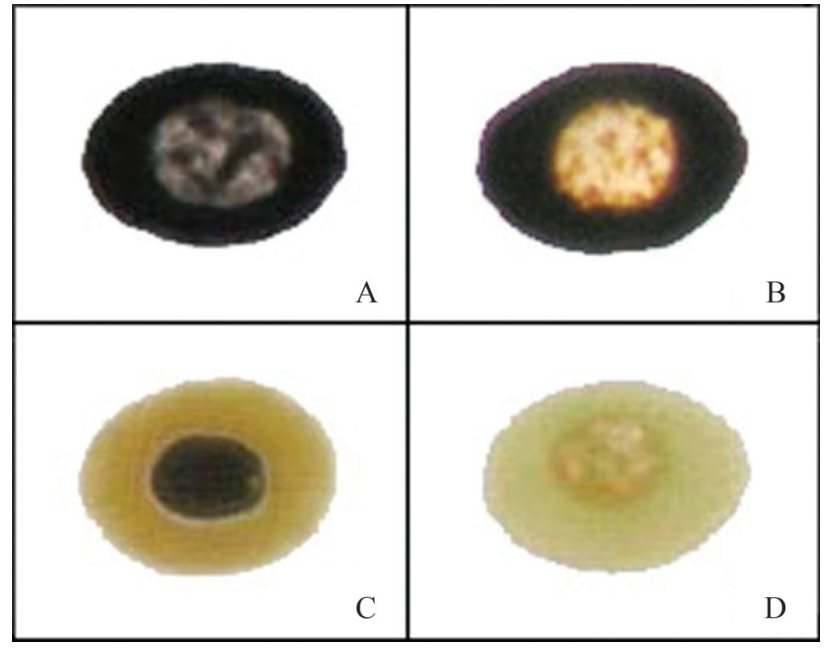

Figure 1 - Differential response of E. johnstonei blood cells to silver staining during the alkaline lysis Comet assay: (A) Cells totally stained, (B) Cells with only cytoplasm stained, (C) Cells with only nuclei stained and (D) Unstained cells.

$\mathrm{AU}_{10 \text { min }}=323 \pm 7, \mathrm{AU}_{15 \text { min }}=326 \pm 9, \mathrm{AU}_{30 \text { min }}=330 \pm 11$ and $\mathrm{AU}_{45 \mathrm{~min}}=361 \pm 5$ ). In this assay, the negative control had a mean arbitrary unit value $\left(\mathrm{AU}_{0 \mathrm{~min}}\right)$ of $38 \pm 5$. In contrast to the enzymatic treatment at $37^{\circ} \mathrm{C}$ overnight (Figure $2 \mathrm{C}$ ), the nuclei were still intact after treatment at $6 \pm 2{ }^{\circ} \mathrm{C}$ during agarose solidification (Figure 2D). Hence, subsequent experiments involving cell lysis were done using only enzymatic treatment at $6 \pm 2{ }^{\circ} \mathrm{C}$. Figure 3 shows images of the Comet categories established for E. johnstonei blood cells.

\section{Estimation of DNA strand breakage in E. johnstonei blood cells}

Table 1 shows the DNA strand breakage induced by BLM and 4NQO in E. johnstonei blood cells at different doses and incubation times. A marked dose-response relationship was observed for both doses and incubation times. Dose-response curves to BLM and 4NQO after a $12 \mathrm{~h}$ exposure (Table 2) showed marked correlations $(\mathrm{R}=0.83$ and $0.90, \mathrm{p} \leq 0.05$; respectively). DNA strand breakage increased significantly ( $\mathrm{p} \leq 0.05$ ) from a concentration of $4.7 \mu \mathrm{g} / \mathrm{mL}$ of BLM and $1.9 \mu \mathrm{M}$ of 4 NQO. The $\% \mathrm{~N}_{\mathrm{Ap} / \mathrm{N}}$ in $E$. johnstonei ranged from $0 \%$ to $8 \%$ (mean: $2.8 \%$ ). The assay reproducibility under the conditions in this model was consistently high, with coefficients of variation $\leq 10 \%$.

\section{Discussion}

In this work, we used a modified Comet assay to detect DNA strand breakage in the direct-developing frog $E$. johnstonei. Previous studies of DNA damage in frogs used alkaline treatment to lyse the cells prior to analysis by the Comet assay (Table 3). Alkaline conditions are generally sufficient to cause cellular lysis in all frog species. 
Eleutherodactylus johnstonei blood cells were resistant to tolerant to alkaline treatment in the standard procedure (Singh et al., 1988). This finding suggests that $E$. johnstonei contains alkali-resistant but proteinase K-sensitive proteins that stabilize and/or protect the nuclei. The Comet assay with enzymatic (proteinase K) lysis has been used to assess DNA intactness in mammalian sperm cells (Baumgartner et al., 2009), which have highly compact nuclear DNA (Ward and Coffey, 1991). Proteinase $\mathrm{K}$ digests proteins associated with nuclei and eliminates DNA-protein bonds generated by some xenobiotics, action that facilitates the electrophoretic migration of damaged DNA (Merk et al., 2000; Singh, 2000b).

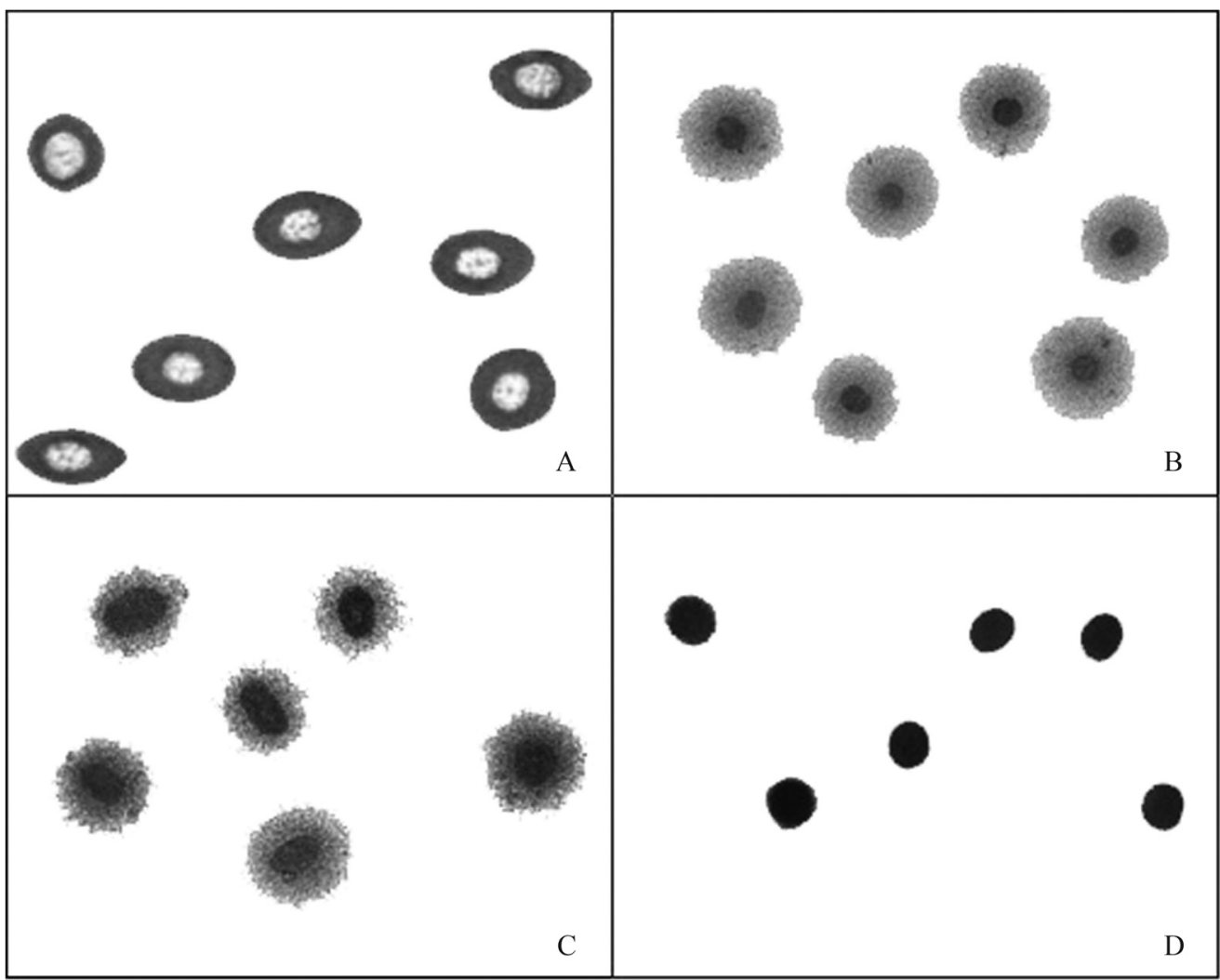

Figure 2 - DNA diffusion assay images from E. johnstonei blood cells after: (A) alkaline lysis, (B) alkaline/enzymatic (40 $\mu \mathrm{g} / \mathrm{mL}$ proteinase $\mathrm{K})$ lysis at $37{ }^{\circ} \mathrm{C}$ overnight, (C) enzymatic $(40 \mu \mathrm{g} / \mathrm{mL}$ proteinase $\mathrm{K})$ lysis at $37^{\circ} \mathrm{C}$ overnight, and (D) enzymatic $(40 \mu \mathrm{g} / \mathrm{mL}$ proteinase $\mathrm{K})$ lysis at $6 \pm 2{ }^{\circ} \mathrm{C}$ during agarose solidification (10 $\mathrm{min})$.
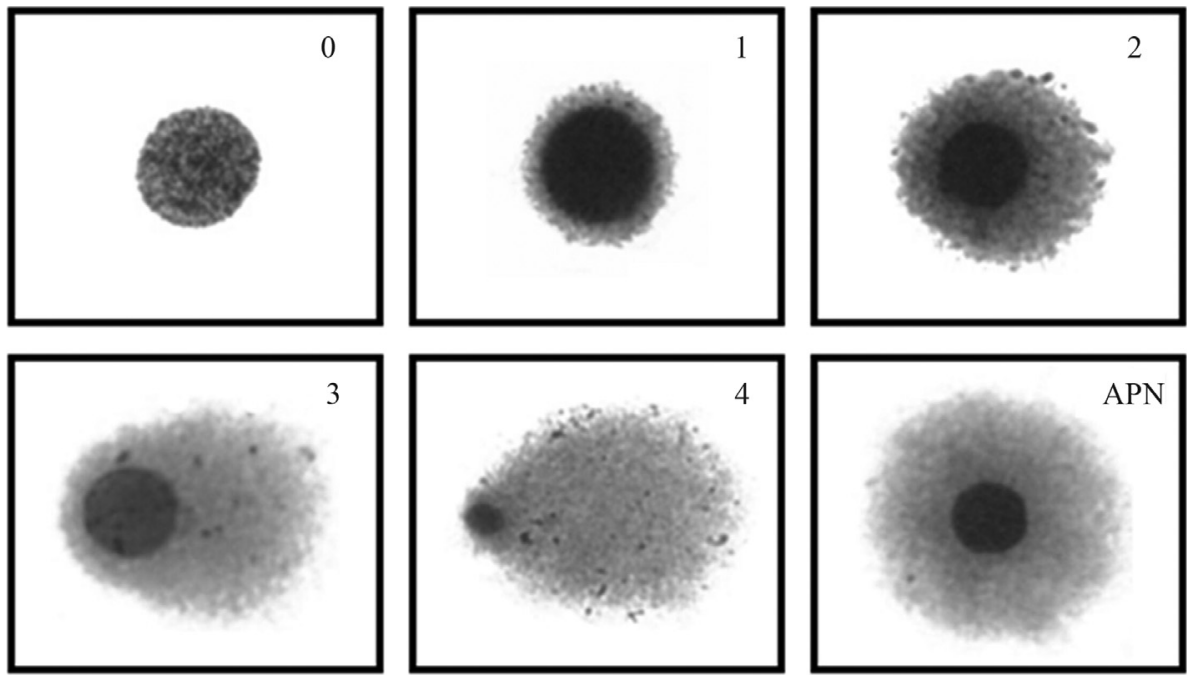

Figure 3 - Visual comet classification into five categories (0-4) proposed by Collins et al. (1997). Images of comets (from E. johnstonei blood cells) stained with silver nitrate. APN: Apoptotic/necrotic nuclei detected by the DNA diffusion assay. 
Table 1 - Estimates of DNA damage in E. johnstonei blood cells exposed to BLM and 4NQO for different times.

\begin{tabular}{|c|c|c|c|c|c|c|c|c|c|c|}
\hline \multirow[t]{3}{*}{ Treatments } & \multicolumn{10}{|c|}{ Leukocyte DNA damage after exposure to mutagens for the indicated times } \\
\hline & \multicolumn{2}{|c|}{$2 \mathrm{~h}(3)^{*}$} & \multicolumn{2}{|c|}{$4 \mathrm{~h}(3)$} & \multicolumn{2}{|c|}{$6 \mathrm{~h}(3)$} & \multicolumn{2}{|c|}{$10 \mathrm{~h}(3)$} & \multicolumn{2}{|c|}{$12 \mathrm{~h}(3)$} \\
\hline & $\mathrm{AU}_{\mathrm{T}}$ & $\mathrm{AU}_{\mathrm{R}}$ & $\mathrm{AU}_{\mathrm{T}}$ & $\mathrm{AU}_{\mathrm{R}}$ & $\mathrm{AU}_{\mathrm{T}}$ & $\mathrm{AU}_{\mathrm{R}}$ & $\mathrm{AU}_{\mathrm{T}}$ & $\mathrm{AU}_{\mathrm{R}}$ & $\mathrm{AU}_{\mathrm{T}}$ & $\mathrm{AU}_{\mathrm{R}}$ \\
\hline $\mathrm{NC}$ & $42 \pm 2$ & $41 \pm 2$ & $41 \pm 0$ & $40 \pm 0$ & $70 \pm 12$ & $68 \pm 12$ & $73 \pm 8$ & $71 \pm 8$ & $72 \pm 9$ & $69 \pm 9$ \\
\hline $\operatorname{BLM}(4.7 \mu \mathrm{g} / \mathrm{mL})$ & $78 \pm 2$ & $77 \pm 2$ & $81 \pm 6$ & $79 \pm 6$ & $114 \pm 12$ & $111 \pm 12$ & $115 \pm 16$ & $112 \pm 16$ & $159 \pm 13$ & $151 \pm 13$ \\
\hline $\operatorname{BLM}(9.5 \mu \mathrm{g} / \mathrm{mL})$ & $82 \pm 2$ & $81 \pm 2$ & $92 \pm 2$ & $90 \pm 2$ & $164 \pm 16$ & $159 \pm 16$ & $149 \pm 10$ & $145 \pm 10$ & $183 \pm 12$ & $174 \pm 12$ \\
\hline $\operatorname{BLM}(19.0 \mu \mathrm{g} / \mathrm{mL})$ & $89 \pm 7$ & $88 \pm 7$ & $110 \pm 3$ & $108 \pm 3$ & $174 \pm 14$ & $169 \pm 14$ & $170 \pm 9$ & $166 \pm 9$ & $219 \pm 7$ & $208 \pm 7$ \\
\hline $\operatorname{BLM}(38.0 \mu \mathrm{g} / \mathrm{mL})$ & $99 \pm 3$ & $98 \pm 3$ & $123 \pm 4$ & $120 \pm 4$ & $172 \pm 9$ & $167 \pm 9$ & $179 \pm 16$ & $174 \pm 16$ & $289 \pm 13$ & $275 \pm 13$ \\
\hline $\operatorname{BLM}(76.0 \mu \mathrm{g} / \mathrm{mL})$ & $101 \pm 5$ & $100 \pm 5$ & $139 \pm 26$ & $137 \pm 26$ & $159 \pm 16$ & $154 \pm 16$ & $209 \pm 7$ & $204 \pm 7$ & $293 \pm 1$ & $279 \pm 1$ \\
\hline $\operatorname{BLM}(152.0 \mu \mathrm{g} / \mathrm{mL})$ & $110 \pm 3$ & $108 \pm 3$ & $164 \pm 15$ & $161 \pm 15$ & $181 \pm 7$ & $176 \pm 7$ & $256 \pm 6$ & $250 \pm 6$ & $332 \pm 8$ & $315 \pm 8$ \\
\hline $\mathrm{NC}$ & $79 \pm 30$ & $79 \pm 30$ & $100 \pm 2$ & $100 \pm 2$ & $91 \pm 16$ & $91 \pm 16$ & $95 \pm 4$ & $95 \pm 4$ & $80 \pm 1$ & $80 \pm 1$ \\
\hline $4 \mathrm{NQO}(1.9 \mu \mathrm{M})$ & $94 \pm 12$ & $93 \pm 12$ & $102 \pm 1$ & $102 \pm 1$ & $115 \pm 2$ & $115 \pm 2$ & $134 \pm 26$ & $134 \pm 26$ & $116 \pm 11$ & $116 \pm 11$ \\
\hline 4NQO $(3.7 \mu \mathrm{M})$ & $92 \pm 8$ & $91 \pm 8$ & $112 \pm 8$ & $112 \pm 8$ & $117 \pm 4$ & $117 \pm 4$ & $142 \pm 1$ & $142 \pm 1$ & $142 \pm 9$ & $142 \pm 9$ \\
\hline 4NQO $(7.5 \mu \mathrm{M})$ & $67 \pm 57$ & $67 \pm 57$ & $116 \pm 8$ & $116 \pm 8$ & $139 \pm 8$ & $139 \pm 8$ & $179 \pm 6$ & $179 \pm 6$ & $184 \pm 17$ & $184 \pm 17$ \\
\hline 4NQO $(15.0 \mu \mathrm{M})$ & $101 \pm 10$ & $100 \pm 10$ & $122 \pm 4$ & $122 \pm 4$ & $146 \pm 11$ & $146 \pm 11$ & $181 \pm 2$ & $181 \pm 2$ & $238 \pm 0$ & $238 \pm 0$ \\
\hline 4NQO $(30.0 \mu \mathrm{M})$ & $97 \pm 2$ & $96 \pm 2$ & $133 \pm 5$ & $133 \pm 5$ & $153 \pm 9$ & $153 \pm 9$ & $193 \pm 0$ & $193 \pm 0$ & $270 \pm 5$ & $270 \pm 5$ \\
\hline $4 \mathrm{NQO}(60.0 \mu \mathrm{M})$ & $102 \pm 3$ & $102 \pm 3$ & $128 \pm 3$ & $128 \pm 3$ & $162 \pm 9$ & $162 \pm 9$ & $196 \pm 3$ & $196 \pm 3$ & $308 \pm 22$ & $308 \pm 22$ \\
\hline
\end{tabular}

The values are the mean \pm SEM from at least three independent experiments with two replicate slides in each. *The total number of male frogs used per experiment with each mutagen. $\mathrm{AU}$ - arbitrary units, $\mathrm{AU}_{\mathrm{T}}$ - total DNA damage measured with the Comet assay, $\mathrm{AU}_{\mathrm{R}}$ - the remaining non-apoptotic/necrotic DNA damage, BLM - bleomycin, 4NQO - 4-nitroquinoline-1-oxide and $\mathrm{NC}$ - negative control $\left(0.9 \% \mathrm{NaCl}\right.$ solution). The $\% \mathrm{~N}_{\mathrm{Ap} / \mathrm{N}}(\mathrm{see}$ Materials and Methods) was the percentage of apoptotic/necrotic nuclei counted in 100 slide fields, and ranged from $0 \%$ to $8 \%$.

Table 2 - Dose-response relationships between BLM and 4NQO concentrations and the estimated DNA damage. An exposure time of $12 \mathrm{~h}$ was used in all experiments.

\begin{tabular}{|c|c|c|c|c|c|c|c|c|c|}
\hline \multirow[t]{3}{*}{ Treatment } & \multicolumn{9}{|c|}{ Leukocyte DNA damage after exposure to mutagen } \\
\hline & \multicolumn{2}{|c|}{ Exp. $1(3)$} & \multicolumn{2}{|c|}{ Exp. 2 (3) } & \multicolumn{2}{|c|}{ Exp. 3 (3) } & \multicolumn{2}{|c|}{ Mean \pm SEM } & \multirow[t]{2}{*}{$\mathrm{CV}(\%)$} \\
\hline & $\mathrm{AU}_{\mathrm{T}}$ & $\mathrm{AU}_{\mathrm{R}}$ & $\mathrm{AU}_{\mathrm{T}}$ & $\mathrm{AU}_{\mathrm{R}}$ & $\mathrm{AU}_{\mathrm{T}}$ & $\mathrm{AU}_{\mathrm{R}}$ & $\mathrm{AU}_{\mathrm{T}}$ & $\mathrm{AU}_{\mathrm{R}}$ & \\
\hline $\mathrm{NC}$ & $72 \pm 9$ & $69 \pm 9$ & $87 \pm 8$ & $80 \pm 8$ & $65 \pm 6$ & $62 \pm 6$ & $75 \pm 9$ & $70 \pm 9$ & 10 \\
\hline $\operatorname{BLM}(4.7 \mu \mathrm{g} / \mathrm{mL})$ & $159 \pm 13 *$ & $151 \pm 13 *$ & $142 \pm 16^{*}$ & $131 \pm 16^{*}$ & $145 \pm 10 *$ & $139 \pm 10 *$ & $149 \pm 7$ & $140 \pm 7$ & 6 \\
\hline $\operatorname{BLM}(9.5 \mu \mathrm{g} / \mathrm{mL})$ & $183 \pm 12 *$ & $174 \pm 12 *$ & $171 \pm 16^{*}$ & $157 \pm 16 *$ & $183 \pm 10 *$ & $176 \pm 10 *$ & $179 \pm 6$ & $169 \pm 6$ & 4 \\
\hline $\operatorname{BLM}(19.0 \mu \mathrm{g} / \mathrm{mL})$ & $219 \pm 7 *$ & $208 \pm 7 *$ & $220 \pm 4 *$ & $202 \pm 4 *$ & $240 \pm 13 *$ & $231 \pm 13 *$ & $226 \pm 9$ & $213 \pm 9$ & 5 \\
\hline $\operatorname{BLM}(38.0 \mu \mathrm{g} / \mathrm{mL})$ & $289 \pm 13 *$ & $275 \pm 13 *$ & $265 \pm 10 *$ & $244 \pm 10 *$ & $276 \pm 14 *$ & $265 \pm 14 *$ & $277 \pm 10$ & $261 \pm 10$ & 4 \\
\hline $\operatorname{BLM}(76.0 \mu \mathrm{g} / \mathrm{mL})$ & $293 \pm 1 *$ & $279 \pm 1 *$ & $299 \pm 3 *$ & $275 \pm 3 *$ & $296 \pm 19 *$ & $285 \pm 19 *$ & $296 \pm 2$ & $279 \pm 2$ & 1 \\
\hline \multirow[t]{2}{*}{$\operatorname{BLM}(152.0 \mu \mathrm{g} / \mathrm{mL})$} & $332 \pm 8 *$ & $315 \pm 8 *$ & $338 \pm 9 *$ & $311 \pm 9 *$ & $343 \pm 3 *$ & $330 \pm 3 *$ & $338 \pm 4$ & $318 \pm 4$ & 2 \\
\hline & & & & & & & \multicolumn{2}{|c|}{$\mathrm{r}=0.83(\mathrm{p} \leq 0.05)$} & \\
\hline $\mathrm{NC}$ & $78 \pm 7$ & $78 \pm 7$ & $80 \pm 1$ & $80 \pm 1$ & $93 \pm 6$ & $93 \pm 6$ & $84 \pm 7$ & $84 \pm 7$ & 10 \\
\hline 4NQO $(1.9 \mu \mathrm{M})$ & $133 \pm 6 *$ & $133 \pm 6 *$ & $116 \pm 11 *$ & $116 \pm 11 *$ & $129 \pm 3 *$ & $129 \pm 3 *$ & $126 \pm 7$ & $126 \pm 7$ & 7 \\
\hline 4NQO $(3.7 \mu \mathrm{M})$ & $147 \pm 2 *$ & $147 \pm 2 *$ & $142 \pm 9 *$ & $142 \pm 9 *$ & $155 \pm 6^{*}$ & $155 \pm 6^{*}$ & $148 \pm 5$ & $148 \pm 5$ & 4 \\
\hline 4NQO $(7.5 \mu \mathrm{M})$ & $176 \pm 16 *$ & $176 \pm 16 *$ & $184 \pm 17 *$ & $184 \pm 17 *$ & $190 \pm 3 *$ & $190 \pm 3 *$ & $183 \pm 6$ & $183 \pm 6$ & 4 \\
\hline 4NQO $(15.0 \mu \mathrm{M})$ & $227 \pm 9 *$ & $227 \pm 9 *$ & $238 \pm 0 *$ & $238 \pm 0 *$ & $222 \pm 11 *$ & $222 \pm 11 *$ & $229 \pm 7$ & $229 \pm 7$ & 4 \\
\hline 4NQO $(30.0 \mu \mathrm{M})$ & $261 \pm 2 *$ & $261 \pm 2 *$ & $270 \pm 5 *$ & $270 \pm 5 *$ & $257 \pm 5 *$ & $257 \pm 5 *$ & $263 \pm 5$ & $263 \pm 5$ & 3 \\
\hline \multirow[t]{2}{*}{$4 \mathrm{NQO}(60.0 \mu \mathrm{M})$} & $316 \pm 8 *$ & $316 \pm 8 *$ & $308 \pm 22 *$ & $308 \pm 22 *$ & $299 \pm 21 *$ & $299 \pm 21 *$ & $308 \pm 7$ & $308 \pm 7$ & 3 \\
\hline & & & & & & & \multicolumn{2}{|c|}{$\mathrm{r}=0.90(\mathrm{p} \leq 0.05)$} & \\
\hline
\end{tabular}

The values are the mean \pm SEM from three independent experiments with two replicate slides in each. The total number of male frogs used per experiment with each mutagen is shown in parentheses. The average values from the three experiments are shown. AU - arbitrary units, $\mathrm{AU}_{\mathrm{T}}$ - total DNA damage measured with the Comet assay, $\mathrm{AU}_{\mathrm{R}}$ - the remaining non-apoptotic/necrotic DNA damage, BLM - bleomycin, $\mathrm{CV}$ - coefficient of variation (\%), 4NQO 4-nitroquinoline-1-oxide, $\mathrm{NC}$ - negative control $\left(0.9 \% \mathrm{NaCl}\right.$ solution) and $\mathrm{r}$ - Pearson correlation coefficient. The $\% \mathrm{~N}_{\mathrm{Ap} / \mathrm{N}}$ (see Materials and Methods) was the percentage of apoptotic/necrotic nuclei counted in 100 slide fields, and ranged from $0 \%$ to $8 \%$. ${ }^{*} \mathrm{p}<0.05$ compared to the negative control (NC) (ANOVA followed by Student's $t$-test). 
Table 3 - Cellular lysis conditions used in different studies to detect DNA damage by the Comet assay in frogs.

\begin{tabular}{|c|c|c|c|c|c|c|}
\hline \multirow[t]{2}{*}{ Genetic model $^{*}$} & \multirow[t]{2}{*}{ Cell type } & \multirow[t]{2}{*}{ Buffer composition } & \multicolumn{3}{|c|}{ Lysis conditions } & \multirow[t]{2}{*}{ References } \\
\hline & & & $\mathrm{pH}$ & Temperature & Time & \\
\hline $\begin{array}{l}\text { Anaxyrus americanus } \\
\text { Lithobates catesbeianus } \\
\text { Lithobates clamitans }\end{array}$ & Erythrocytes & $\begin{array}{l}\text { Buffer 1: } 2.5 \mathrm{M} \mathrm{NaCl}, 100 \mathrm{mM} \\
\mathrm{Na}_{2} \mathrm{EDTA}, 10 \mathrm{mM} \text { Tris- } \mathrm{HCl} \text {, } \\
10 \% \text { DMSO, } 1 \% \text { sodium } \\
\text { sarcosinate, pH } 10\end{array}$ & alkaline & Room temperature & $2 \mathrm{~h}$ & $\begin{array}{l}\text { Ralph et al. }(1996) \\
\text { Clements et al. (1997) } \\
\text { Ralph and Petras } \\
\text { (1998a) }\end{array}$ \\
\hline $\begin{array}{l}\text { Anaxyrus americanus } \\
\text { Lithobates clamitans } \\
\text { Euphlyctis hexadactylus } \\
\text { Lithobates pipiens }\end{array}$ & Erythrocytes & $\begin{array}{l}{ }^{\dagger} \text { Buffer 2: } 2.5 \mathrm{M} \mathrm{NaCl}, 100 \mathrm{mM} \\
\mathrm{Na}_{2} \text { EDTA, } 10 \mathrm{mM} \text { Tris- } \mathrm{HCl} \text {, } \\
\text { 10\% DMSO, } 1 \% \text { sodium sarco- } \\
\text { sinate, } 1 \% \text { Triton X- } 100, \mathrm{pH} 10\end{array}$ & alkaline & Room temperature & $2 \mathrm{~h}$ & $\begin{array}{l}\text { Ralph and Petras (1997) } \\
\text { Ralph and Petras } \\
\text { (1998b) } \\
\text { Rajaguru et al. (2001) }\end{array}$ \\
\hline $\begin{array}{l}\text { Fejervarya limnocharis } \\
\text { Pelophylax nigromaculata }\end{array}$ & Erythrocytes & Buffer 2 & alkaline & Room temperature & $1 \mathrm{~h}$ & $\begin{array}{l}\text { Feng et al. (2004) } \\
\text { Liu et al. (2011) }\end{array}$ \\
\hline $\begin{array}{l}\text { Xenopus laevis } \\
\text { Silurana tropicalis }\end{array}$ & Lymphocytes & Buffer 2 & alkaline & On ice $\left(0^{\circ} \mathrm{C}\right)$ & $30 \mathrm{~min}$ & Banner et al. (2006) \\
\hline Pseudepidalea raddei & Hepatocytes & Buffer 2 & alkaline & $4{ }^{\circ} \mathrm{C}$ & $1 \mathrm{~h}$ & Liu et al. (2006) \\
\hline $\begin{array}{l}\text { Bufo gargarizans } \\
\text { Pseudepidalea raddei }\end{array}$ & $\begin{array}{l}\text { Erythrocytes } \\
\text { Hepatocytes }\end{array}$ & Buffer 2 & alkaline & $4^{\circ} \mathrm{C}$ & $2 \mathrm{~h}$ & $\begin{array}{l}\text { Huang et al. }(2007) \\
\text { Yin et al. }(2008) \\
\text { Yin et al. }(2009)\end{array}$ \\
\hline Pelophylax nigromaculata & Testicular cells & $\begin{array}{l}\text { Buffer 3: } 2.5 \mathrm{M} \mathrm{NaCl}, 10 \mathrm{mM} \\
\mathrm{Na}_{2} \text { EDTA, } 10 \mathrm{mM} \text { Tris- } \mathrm{HCl} \text {, } \\
\text { 10\% DMSO, } 1 \% \text { SDS, } 1 \% \text { Tri- } \\
\text { ton X-100, pH } 10\end{array}$ & alkaline & $4^{\circ} \mathrm{C}$ & $2-4 \mathrm{~h}$ & Wang and Jia (2009) \\
\hline $\begin{array}{l}\text { Pelophylax lessonae } \\
\text { Xenopus laevis }\end{array}$ & Erythrocytes & $\begin{array}{l}\text { Buffer 4: } 2.5 \mathrm{M} \mathrm{NaCl}, 100 \mathrm{mM} \\
\mathrm{Na}_{2} \text { EDTA, } 10 \mathrm{mM} \text { Tris-HCl, } \\
\text { 10\% DMSO, } 1 \% \text { Triton } \\
\text { X-100, pH } 10\end{array}$ & alkaline & $4^{\circ} \mathrm{C}$ & $1 \mathrm{~h}$ & $\begin{array}{l}\text { Maselli et al. (2010) } \\
\text { Mouchet et al. (2006) }\end{array}$ \\
\hline $\begin{array}{l}\text { Eleutherodactylus } \\
\text { johnstonei }\end{array}$ & Erythrocytes & $\begin{array}{l}\text { Buffer 5: } 50 \mathrm{mM} \text { Tris- } \mathrm{HCl} \text {, } \\
10 \mathrm{mM} \mathrm{CaCl}_{2}, 0.04 \mathrm{mg} / \mathrm{mL} \\
\text { proteinase } \mathrm{K}, 0.8 \% \mathrm{LGT} \\
\text { agarose, } \mathrm{pH} 8\end{array}$ & neutral & $\begin{array}{l}\text { Kept at } 6 \pm 2{ }^{\circ} \mathrm{C} \text { for } \\
\text { solidification }\end{array}$ & $10 \mathrm{~min}$ & Present work \\
\hline
\end{tabular}

(*)Anuran species names follow Frost (2011). ${ }^{\dagger}$ First reported by Singh et al. (1988).

The intactness of sperm DNA is regularly analyzed with the Comet assay after alkaline and enzymatic treatments (Speit et al., 2009). However, alkaline/enzymatic treatment was particularly aggressive to nuclear stability in E. johnstonei blood cells. For this reason, we used a neutral (pH 8) and single enzymatic digestion with proteinase $\mathrm{K}$ in situ in agarose gels; this procedure considerably reduced the assay costs and time. The temperature during cellular lysis is another critical variable that affects basal DNA damage in the Comet assay, as indicated in previous reports (Speit et al., 1999; Banáth et al., 2001). In E. johnstonei erythrocytes, enzymatic lysis at low temperature $\left(6 \pm 2{ }^{\circ} \mathrm{C}\right)$ was ideal for obtaining naked nuclei with low levels of basal DNA damage.

The results described here show that the Comet assay can provide a good estimation of DNA damage in $E$. johnstonei. The assay was reproducible and sensitive enough to detect DNA strand breakage in E. johnstonei blood cells. The basal DNA damage estimated for the species agreed with previously reported values (Collins et al., 1997). In addition, the DNA damage observed here was poorly associated to apoptosis and necrosis, in contrast to the situation in humans (Tice et al., 2000), sea lions (ElZein et al., 2006) and dolphins (Díaz et al., 2009).

This study has shown the usefulness of amphibians as bio-indicators. A simultaneous study (Meza-Joya et al., in preparation) in our laboratory examined the toxic and genotoxic effects of a glyphosate-based herbicide (Roundup ${ }^{\mathbb{B}} \mathrm{SL}$ - Cosmoflux ${ }^{\circledR} 411 \mathrm{~F}$ ) on E. johnstonei. The study again showed that the Comet assay was highly sensitive for detecting DNA damage induced by this herbicide. This finding suggests that the Comet assay is an accurate method for detecting DNA damage in E. johnstonei after exposure to environmental xenobiotics.

In conclusion, the alkaline Comet assay (Singh et al., 1988) was inappropriate for measuring DNA strand breakage in E. johnstonei. Alkaline lysis can be replaced by enzymatic lysis (proteinase K), with good results. In contrast, combined alkaline/enzymatic treatment or long incubations (overnight) at $37{ }^{\circ} \mathrm{C}$ with proteinase $\mathrm{K}$ generate unstable nuclei and result in consistently elevated basal DNA damage. The contribution of apoptosis and necrosis to the overall DNA damage in E. johnstonei was negligible, as assessed by the Comet assay. The Comet assay is a repro- 
ducible, sensitive method for detecting DNA strand breakage in E. johnstonei.

\section{Acknowledgments}

The Corporación Regional para la Defensa de la Meseta de Bucaramanga (CDMB) provided the research and collecting permits. This work was supported by the Vicerrectoría de Investigaciones y Extensión, Universidad Industrial de Santander, Colombia (Grant No. 5163).

\section{References}

Banáth JP, Kim A and Olive PL (2001) Overnight lysis improves the efficiency of detection of DNA damage in the alkaline Comet assay. Radiat Res 155:564-571.

Banner SH, Ruben LN and Johnson RO (2006) Bleomycin-induced DNA damage and repair in Xenopus laevis and Xenopus tropicalis. J Exp Zool 307A:84-90.

Baohong W, Jiliang H, Lifen J, Deqiang L, Wei Z, Jianlin L and Hongpin D (2005) Studying the synergistic damage effects induced by $1.8 \mathrm{GHz}$ radiofrequency field radiation (RFR) with four chemical mutagens on lymphocyte DNA using comet assay in vitro. Mutat Res 578:149-157.

Baumgartner A, Cemeli E and Anderson D (2009) The Comet assay in male reproductive toxicology. Cell Biol Toxicol 25:81-98.

Berrill M, Bertram S, McGillivray L, Kolohon M and Paull B (1994) Effects of low concentrations of forest-use pesticides on frog embryos and tadpole. Environ Toxicol Chem 13:657-664.

Blaustein AR and Wake DB (1990) Declining amphibian populations: a global phenomenon. Trends Ecol Evol 5:203-204.

Clements C, Ralph S and Petras M (1997) Genotoxicity of select herbicides in Rana catesbeiana tadpoles using the alkaline single-cell gel DNA electrophoresis (Comet) assay. Environ Mol Mutagen 29:277-288.

Collins AR (2002) The comet assay, principles, applications and limitations. In: Didenko VV (ed.) In Situ Detection of DNA Damage. Methods and Protocols, v. 203. Humana Press Inc., Totowa, pp 163-177.

Collins AR, Dusinska A, Franklin M, Somorovska M, Petrovska H, Duthie S, Fillion L, Panayoitidis M, Raslova K and Vaughan N (1997) Comet assay in human biomonitoring studies: reliability, validation and applications. Environ Mol Mutagen 30:139-146.

Cotelle S and Férard JF (1999) Comet assay in genetic ecotoxicology: a review. Environ Mol Mutagen 34:246-255.

Dhawan A, Bajpayee M and Parmar D (2009) Comet assay: a reliable tool for the assessment of DNA damage in different models. Cell Biol Toxicol 25:5-32.

Díaz A, Carro S, Santiago L, Estévez J, Guevara C, Blanco M, Sánchez L, Sánchez L, López N, Cruz D, et al. (2009) Estimates of DNA strand breakage in bottlenose dolphin (Tursiops truncatus) leukocytes measured with the Comet and DNA diffusion assays. Genet Mol Biol 32:367-372.

El-Zein RA, Hastings-Smith DA, Ammenheuser MM, TrinenMoslen M, Gulland FM and Ward JB (2006) Evaluation of two different biomarkers for use in the assessment of toxic chemical exposure in California sea lions (Zalophus californianus). Marine Pollut Bull 52:104-120.
Feng S, Kong Z, Wang X, Zhao L and Peng P (2004) Acute toxicity and genotoxicity of two novel pesticides on amphibian, Rana n. hallowell. Chemosphere 56:457-463.

Hartmann A, Agurell E, Beevers C, Brendler-Schwaab S, Burlinson B, Clay P, Collins A, Smith, A, Speit G, Thybaud V, et al. (2003) Recommendations for conducting the in vivo alkaline Comet assay. Mutagenesis 18:45-51.

Hedges SB, Duellman WE and Heinicke MP (2008) New World direct-developing frogs (Anura, Terrarana): Molecular phylogeny, classification, biogeography, and conservation. Zootaxa 1737:1-182.

Huang D, Zhang Y, Wang Y, Xie Z and Ji W (2007) Assessment of the genotoxicity in toad Bufo raddei exposed to petrochemical contaminants in Lanzhou region, China. Mutat Res 629:81-88.

Kaiser H (1997) Origins and introductions of the Caribbean frog, Eleutherodactylus johnstonei (Leptodactylidae): Management and conservation concerns. Biodiv Conserv 6:13911407.

Kaiser H, Barrio-Amorós CL, Trujillo JD and Lynch JD (2002) Expansion of Eleutherodactylus johnstonei in northern South America: Rapid dispersal through human interactions. Herpetol Rev 33:290-294.

Kumaravel TS and Jha AN (2006) Reliable comet assay measurements for detecting DNA damage induced by ionizing radiation and chemicals. Mutat Res 605:7-16.

Kumaravel TS, Vilhar B, Faux SP and Jha AN (2009) Comet assay measurements: A perspective. Cell Biol Toxicol 25:5364.

Lips KR (1998) Decline of a tropical montane amphibian fauna. Conserv Biol 12:106-112.

Liu WY, Wang CY, Wang TS, Feller GM, Lai BC and Kam YC (2011) Impacts of the herbicide butachlor on the larvae of a paddy field breeding frog (Fejervarya limnocharis) in subtropical Taiwan. Ecotoxicology 20:377-384.

Liu Y, Zhang Y and Huang D (2006) The role of reactive oxygen species in the herbicide acetochlor-induced DNA damage on Bufo raddei tadpole liver. Aquat Toxicol 78:21-26.

Maselli V, Polese G, Rippa D, Ligrone R, Rastogi RK and Fulgione D (2010) Frogs, sentinels of DNA damage induced by pollution in Naples and the neighbouring provinces. Ecotoxicol Environ Saf 73:1525-1529.

Merk O, Reiser K and Speit G (2000) Analysis of chromateinduced DNA-protein crosslinks with the comet assay. Mutat Res 471:71-80.

Mouchet F, Gauthier L, Mailhes C, Jourdain MJ, Ferrier V, Triffault G and Devaux A (2006) Biomonitoring of the genotoxic potential of aqueous extracts of soils and bottom ash resulting from municipal solid waste incineration, using the Comet and micronucleus tests on amphibian (Xenopus laevis) larvae and bacterial assays (Mutatox and Ames tests). Sci Total Environ 355:232-246.

Nigro M, Frenzilli G, Scarcelli V, Gorbi S and Regoli F (2002) Induction of DNA strands breakage and apoptosis in the eel Anguilla anguilla. Marine Environ Res 54:517-520.

Ortega JE, Jerez A and Ramírez-Pinilla MP (2001) Eleutherodactylus johnstonei. Herpetol Rev 32:269.

Ortega JE, Serrano VH and Ramírez-Pinilla MP (2005) Reproduction of an introduced population of Eleutherodactylus johnstonei at Bucaramanga, Colombia. Copeia 2005:642648. 
Rajaguru P, Kalpana R, Hema A, Suba S, Baskarasethupathi B, Kumar PA and Kalaiselvi K (2001) Genotoxicity of some sulfur dyes on tadpoles (Rana hexadactyla) measured using the Comet assay. Environ Mol Mutag 38:316-322.

Ralph S and Petras M (1997) Genotoxicity monitoring of small bodies of water using two species of tadpoles and the alkaline single cell gel (Comet) assay. Environ Mol Mutag 29:418-430.

Ralph S and Petras M (1998a) Comparison of sensitivity to methyl methanesulphonate among tadpole developmental stages using the alkaline single cell gel DNA electrophoresis (Comet) assay. Environ Mol Mutag 31:374-382.

Ralph S and Petras M (1998b) Caged amphibian tadpoles and in situ genotoxicity monitoring of aquatic environments with the alkaline single cell gel electrophoresis (Comet) assay. Mutat Res 413:235-250.

Ralph S, Petras M, Pandrangi R and Vrzoc M (1996) Alkaline single-cell gel (Comet) assay and genotoxicity monitoring using two species of tadpoles. Environ Mol Mutag 28:112120.

Relyea RA (2004a) Synergistic impacts of Malathion and predatory stress on six species of North American tadpoles. Environ Toxicol Chem 23:1080-1084.

Relyea RA (2004b) Grown and survival of five amphibian species exposed to combinations of pesticides. Environ Toxicol Chem 23:1735-1742.

Rödder D (2009) Human footprint, facilitated jump dispersal, and the potential distribution of the invasive Eleutherodactylus johnstonei Barbour 1914 (Anura, Eleutherodactylidae). Trop Zool 22:205-217.

Singh NP (2000a) A simple method for accurate estimation of apoptotic cells. Exp Cell Res 256:328-337.

Singh NP (2000b) Microgels for estimation of DNA strand breaks, DNA protein crosslinks and apoptosis. Mutat Res 455:111-127.

Singh NP, McCoy MT, Tice RR and Schneider EL (1988) A simple technique for quantitation of low levels of DNA damage in individual cells. Exp Cell Res 175:184-191.
Speit G, Trenz K, Schütz P, Rothfuss A and Merk O (1999) The influence of temperature during alkaline treatment and electrophoresis on results obtained with the Comet assay. Toxicol Lett 110:73-78.

Speit G, Vasquez M and Hartman A (2009) The Comet assay as an indicator test for germ cell genotoxicity. Mutat Res 681:312.

Tice RR, Agurell E, Anderson D, Burlinson B, Hartmann A, Kobayashi H, Miyamae Y, Rojas E, Ryu JC and Sasaki YF (2000) Single cell gel/Comet assay: guidelines for in vitro and in vivo genetic toxicology testing. Environ $\mathrm{Mol}$ Mutagen 35:206-221.

Wang MZ and Jia XY (2009) Low levels of lead exposure induce oxidative damage and DNA damage in the testes of the frog Rana nigromaculata. Ecotoxicology 18:94-99.

Ward WS and Coffey DS (1991) DNA packaging and organization in mammalian spermatozoa: Comparison with somatic cells. Biol Reprod 44:569-574.

Yin XH, Li SN, Zhang L, Zhu GN and Zhuang HS (2008) Evaluation of DNA damage in Chinese toad (Bufo bufo gargarizans) after in vivo exposure to sublethal concentration of four herbicides using the comet assay. Ecotoxicology 17:280-286.

Yin XH, Zhu GN, Li XB and Liu SY (2009) Genotoxicity evaluation of chlopyrifos to amphibian Chinese toad (Amphibian, Anura) by Comet assay and micronucleus test. Mutat Res 680:2-6.

\section{Internet Resources}

Frost DR (2011) Amphibian Species of the World: An Online Reference, v. 5.5. Electronic Database accessible at http://research.amnh.org/vz/herpetology/amphibia/. American Museum of Natural History, New York, USA (Accessed on February 24, 2011).

Associate Editor: Daisy Maria F. Salvadori

License information: This is an open-access article distributed under the terms of the Creative Commons Attribution License, which permits unrestricted use, distribution, and reproduction in any medium, provided the original work is properly cited. 